\title{
Direct numerical simulations of bubbly flows
}

\author{
Gretar TRYGGVASON* and Jiacai LU* \\ *University of Notre Dame \\ Department of Aerospace and Mechanical Engineering, Notre Dame, IN, 46556, USA \\ E-mail: gtryggva@nd.edu
}

Received 2 April 2015

\begin{abstract}
Direct numerical simulations (DNS) of multi-fluid and multiphase flows have progressed enormously over the last decade or two. It is, in particular, now possible to simulate the evolution of hundreds of bubbles in laminar and turbulent flows for a long enough time so that meaningful statistical quantities can be collected. For bubbly flow in vertical channels DNS have provided considerable new insight into the structure of the flow and how it can be modeled. The flow structure depends sensitively on the sign of the lift force on the bubbles. For nearly spherical bubbles in both upflow and downflow the lateral migration of bubbles results in a core region where the weight of the mixture exactly balances the imposed pressure gradient. For upflow bubbles accumulate at the wall but for downflow the region next to the wall is free of bubbles. The results lead to a very simple model of the void fraction distribution and, for downflow the velocity and the flow rate can be predicted relatively accurately. Deformable bubbles result in a very different flow structure, with no bubbles accumulating at the wall. Simulations of the transient motion show that it takes a long time for the flow to reach a steady state and that the evolution is complex, with bubbles moving in and out of the wall-layer. The availability of DNS results calls for more intense efforts to use the data for developing closure terms for models of the average and large-scale flows, as well as the development of efficient and accurate methods for more complex flows, such as those undergoing topology changes and involving additional physical effects like surfactants and heat and mass transfer.
\end{abstract}

Key words: Multiphase flow, Direct numerical simulations, Turbulent bubbly channel flows, Analytical models

\section{Introduction}

Bubbly flows are found in a large number of industrial processes and natural phenomena (Furusaki et al., 2001); Deckwer, 1992). Common examples include the processing of a large number of industrial chemicals in bubble columns, the generation of vapor in boilers, cavitation bubbles in diverse marine applications, air entrainment resulting from wave breaking at the ocean/atmosphere interface, and degassing in magma during volcanic eruptions. An understanding of bubbly flows and, in particular, the ability to accurately predict the behavior of such flows is therefore of profound importance. A fully predictive capability would not only allow us to optimize the performance of a large number of processes relying on bubbly flows, but also allow the development of new processes.

Scientific inquiry into bubble behavior has a long history, including analytical solutions for the rise of a single incompressible bubble and oscillations of compressible ones. Experiments have also played a major role and contributed both understanding and the ability to design devices relying on bubbly flows. The experimental challenges are, however, significant. Not only are dense bubbly flows often optically inaccessible, but experimentally it can be difficult to control the exact setup, such as to ensure monodispere mixture and, of course, sometimes there are physical effects, such as surfactants, that are present but not understood sufficiently well so that they can be accounted for in theoretical models. The observation that experimental results matched the solution for a solid sphere rather than a clean bubble, and the realization that surfactants usually make the surface immobile, is perhaps the best demonstration of the importance of supplementing experiments by mathematical descriptions. Unfortunately, the motion of a deformable 
bubble rising at finite Reynolds numbers is, for all practical purposes, unsolvable analytically. The motion of many bubbles is even more intractable. In the last few decades the ability to conduct direct numerical simulations (DNS), where all continuum scales are resolved accurately for systems containing sufficiently large number of bubbles and range of flow scales to allow non-trivial interactions of different scales, has provided the ability to examine very well-defined systems where every quantity of interest can be recorded and examined.

Bubbly flows occur in many circumstances, but the collective rise of many buoyant bubbles, both in unbounded domains and channels, has particularly broad applications and is therefore of a major interest. Here, we discuss relatively recent studies where DNS is used to follow the evolution of bubbly flows in vertical channels. Such flows have been examined experimentally by a number of investigators and while a complete review of experimental studies is beyond the scope of the present review, we note the early study by Serizawa et al. (1975a, 1975b), where the void fraction and velocity profiles in turbulent air-water bubbly channel flows were examined, as well as more recent studies by Wang et al. (1987); Liu and Bankoff (1993); Nakoryakov et al. (1996); Liu (1997); Kashinsky and Randin (1999); So et al. (2002); Guet et al. (2004); Matos et al. (2004); Zenit et al. (2004); Descamps et al. (2008); and Mendez-Diaz et al. (2012). While the results obtained by different investigators sometimes differ slightly, probably due to different bubble sizes, they all agree that for nearly spherical bubbles the void fraction distribution and the velocity profile in the core of the channel are relatively uniform and that a void fraction peak is generally found near the wall for upflow but not for downflow. Large and deformable bubbles, on the other hand, show the opposite behavior and do not accumulate at the walls in upflow. A number of authors have also developed two-fluid models for the average bubbly flows in channels. Antal et al. (1991) and Azpitarte and Buscaglia (2003) solved a two fluid model of laminar bubbly flow analytically, and Drew and Lahey (1993) solved equations for turbulent flows using asymptotic analysis. The models reproduced the general trends seen experimentally, including uniform velocity and void fraction in the core, wall peaking for upflow and a wall-layer without bubbles for downflow, but require the adjustments of closure terms for the unresolved scales. Numerical solutions of the two-fluid equations include, for example, Lopez De Bertodano et al. (1987, 1994); Kuo et al. (1997), and Guet et al. (2005), as well as Celik and Gel (2002) and Politano et al. (2003) who extended the models to non-uniform bubble distributions. In addition, several authors - such as Molin et al. (2012) - have simulated the liquid flow using DNS and modeled the bubbles as point particles that are coupled to the liquid through empirical or semi-empirical correlations.

\section{Numerical Methods}

Computations of multiphase flows go back to the beginning of computational fluid dynamics. Indeed, although today we often refer to a projection method on a staggered grid as a MAC method, the M in the Marker-And-Cell (MAC) method of Harlow and collaborators (Harlow and Welch, 1965) stood for the markers used to identify different fluids. The markers were soon replaced by a marker function advected by the Volume-Of-Fluid method (Hirt and Nichols, 1981) and while the results were generally superior to those of the MAC method, early results show considerable irregularities at the interface between the different fluids. In the eighties and early nineties better advection methods (Youngs, 1982) and new ways to find surface tension (Brackbill et al., 1992) improved the results obtained by VOF methods, and alternative methods were also developed. The Level Set method (Sussman et al. 1994) quickly became very popular, in part because of its simplicity and in part because the original method had significant room for improvements. The early nineties also saw the introduction of the front-tracking method of Unverdi and Tryggvason (1992), where the interface is represented by connected marker points, or a front. The marker points are advected by the flow and the marker function is constructed from the location of the front.

The MAC and the VOF methods were implemented on regular structured grids using a single set of equations to represent both fluids. Today this is by far the most common strategy and it is used in, for example, most implementations of the level set method and the front tracking method of Unverdi and Tryggvason (1992). In the one-fluid formulation the Navier-Stokes equations, which are valid for the whole domain and incorporate the jump conditions at the interfaces, are

$$
\rho \frac{\partial \mathbf{u}}{\partial t}+\rho \nabla \cdot \mathbf{u} \mathbf{u}=-\nabla p+\rho \mathbf{g}+\nabla \cdot \mu\left(\nabla \mathbf{u}+\nabla \mathbf{u}^{T}\right)+\sigma \int_{F} \kappa_{f} \mathbf{n}_{f} \delta\left(\mathbf{x}-\mathbf{x}_{f}\right) d A_{f}
$$

Here, $\mathbf{u}$ is the velocity vector, $p$ is the pressure, and $\rho$ and $\mu$ are the discontinuous density and viscosity fields, 
respectively, $\mathbf{g}$ is the gravity acceleration, $\sigma$ is the constant surface tension, and $\delta$ is a three-dimensional delta function constructed by repeated multiplication of one-dimensional delta functions. $\kappa_{\mathrm{f}}$ is twice the mean curvature, $\mathbf{n}_{\mathrm{f}}$ is a unit vector normal to the front, $\mathbf{x}$ is the point at which the equation is evaluated, and $\mathbf{x}_{\mathrm{f}}$ is the position of the front. Because of the incompressibility of the liquid and bubbles, the mass conservation equation reduces to

$$
\nabla \cdot \mathbf{u}=0
$$

for the entire domain. When combined with the momentum equation, Eq. (2) leads to a nonseparable elliptic equation for the pressure. In most of the simulations discussed here, the density and viscosity of each fluid are taken to be constants.

The one-fluid Navier-Stokes equations can be solved by essentially any standard method used for single-phase flows, but in many - if not most - applications, a projection method implemented using a regular structured staggered mesh is used. The difference between the different approaches is in how the marker function, identifying the different fluids is advected. For the results presented here, a front tracking method is used. The method was originally introduced by Unverdi and Tryggvason (1992), and various updates, improvements and accuracy tests can be found in Tryggvason et al. (2001, 2011). A comparison between the front tracking method and a Lattice Boltzman Method (LBM) showed that results for the rise of a single bubble are in good agreement (Sankaranarayanan et al., 2003). For other implementation of the method described here and applications to bubbly flows, see Dijkhuizen et al. (2010a, 2010b); van Sint Annaland et al. (2006); Hao and Prosperetti (2004); Hua and Lou (2007); and Muradoglu and Kayaalp (2006), for example.

\section{Clean Bubbles in Vertical Channels}

Early DNS studies of bubbly flows focused on fully periodic domains, with applications to homogenous flows. The results showed that the behavior of freely moving and interacting bubbles was generally different from cell-models where the bubbles were held fixed in a periodic array, but that low order statistics generally converged quickly as the number of bubbles per periodic domain was increased. The results also showed that the bubble deformation has an important effect on the microstructure of the mixture. Nearly spherical bubbles, for example, generally show a tendency to pair up horizontally, increasing the drag and slowing the rise velocity, whereas deformable bubbles tend to line up vertically, one behind the other, and since this reduced the drag the rise velocity increases. Extensive discussions of other results obtained from the DNS data, such as more details about the microscopic bubble distribution and interactions, pseudo turbulence, and the dispersion of bubbles with time, can be found in Esmaeeli and Tryggvason (1998, 1999, 2005) and Bunner and Tryggvason (2002a,b, 2003).

DNS of many bubbles of the same size in vertical channels have shown that once the flow reaches a statistically steady state, its structure is relatively simple and completely governed by the direction of the lift force on the bubbles. Nearly spherical bubbles move laterally from regions of low relative velocity to regions of high relative velocity. For upflow in a vertical channel the bubbles are therefore driven to the walls, out of the core region of the channel, and for downflow they move away from the walls, into the core region. In upflow this motion results in an increased density of the core region and for high enough void fraction the migration of bubbles continues until the weight of the mixture is balanced by the imposed pressure gradient. When those are in balance the average shear is reduced to zero and the migration of bubbles stops. For downflow the bubble migration to the core reduces the weight of the fluid mixture there, or increases its buoyancy, such that the weight eventually balances the pressure gradient that drives the motion downward. The average fluid shear again is reduced to zero and the bubble migration stops. Thus, for both upflow and downflow the migration of bubbles results in core regions whose density is such that it matches the imposed pressure gradient, and the void fraction is easily calculated, as shown by Lu et al. (2006). At steady state a force balance on an elementary control volume in the channel results in:

$$
\frac{d}{d x} \tau(x)-\frac{d p}{d y}-\rho(x) g=0 .
$$

Here, $\tau(x)$ is the shear stress, the second term is the imposed pressure gradient and the last term is the weight of the mixture. The sum of the pressure gradient and the weight of the mixture, or the sign of $\beta=d p / d y+\rho_{a v} g$, determines the flow direction. For $\beta<0$ the pressure gradient overcomes the weight of the mixture and the flow is upward, but for $\beta>0$ the flow is downward. For both upflow and downflow the wall shear must balance the net pressure gradient 
at steady state:

$$
2 \tau_{w}=-\beta W
$$

where $W$ is the width of the channel. This relationship, together with a constant flow rate, can be used to check whether the flow has reached a steady state. The local density is a function of the void fraction, $\rho=\varepsilon \rho_{g}+(1-\varepsilon) \rho_{l}$, and the average channel density, $\rho_{a v}$, and the average channel void fraction, $\varepsilon_{a v}$, are related by $\rho_{a v}=\varepsilon_{a v} \rho_{g}+\left(1-\varepsilon_{a v}\right) \rho_{l}$. Thus, (3) becomes:

$$
\frac{d}{d x} \tau(x)-\beta-g \Delta \rho\left(\varepsilon_{a v}-\varepsilon(x)\right)=0
$$

where $\Delta \rho=\rho_{l}-\rho_{g}$ is the density difference. If the weight of the mixture matches the imposed pressure gradient, the shear must be zero and the bubble migration stops. Assuming that at steady state this is the case in the core region of the channel, the void fraction there must be:

$$
\varepsilon_{c}=\varepsilon_{a v}+\frac{\beta}{g \Delta \rho} .
$$

For upflow, where $\beta<0$, the void fraction in the center will therefore be less than $\varepsilon_{a v}$, but for downflow, where $\beta>0$, the void fraction in the center will be larger than $\varepsilon_{a v}$. If the pressure gradient is sufficiently large for upflow, all the bubbles are driven to the walls and the void fraction in the center becomes zero. The discussion below is limited to upflow cases where $\varepsilon_{a v}+\beta / g \Delta \rho>0$. For downflow, the only limitation is that the void fraction in the center cannot be larger than unity, although in practice the assumption of small spherical bubbles is likely to break down at high void fractions.

For downflow, the bubbles that increase the void fraction in the center come from the walls, resulting in a bubble free wall-layer, whose thickness can be found by mass conservation:

$$
\varepsilon_{a v} W=\varepsilon_{c}(W-2 \delta) \text {. }
$$

Substituting for the void fraction in the core region gives the wall-layer thickness:

$$
\delta=\frac{\beta W}{2\left(\beta+\varepsilon_{a v} g \Delta \rho\right)} .
$$

Near the walls there are no bubbles, so the shear is given by $\tau=\mu_{l}(d v / d x)$ and the velocity is easily computed, particularly for laminar flow. The flow is parallel, bounded by the wall on one side and the shear free core region on the other, and is found by integrating

$$
\mu_{l} \frac{d^{2} v}{d x^{2}}=\beta+\Delta \rho g \varepsilon_{a v},
$$

using the boundary conditions that $v=0$ at $x=0$ and $d v / d x=0$ at $x=\delta$. The velocity in the wall-layer is therefore:

$$
v(x)=\frac{\left(\beta+\varepsilon_{a v} g \Delta \rho\right)}{2 \mu_{l}}\left(x^{2}-2 \delta x\right) .
$$

The velocity in the middle is uniform and can be found by taking $x=\delta$ in equation (10):

$$
v_{c}=\frac{-\beta^{2} W^{2}}{8 \mu_{l}\left(\beta+\varepsilon_{a v} g \Delta \rho\right)} .
$$

The velocity in the wall-layer determines the constant velocity in the core region and the total flow rate in bubbly downflow is easily computed. For laminar flow the volume flux in the wall-layer is found by integrating equation (10), resulting in

$$
Q_{\delta}=\int_{0}^{\delta} v_{l}(x) d x=-\left(\beta+\varepsilon_{a v} g \Delta \rho\right) \delta^{3} / 3 \mu
$$

since there are no bubbles there. The total liquid volume flux is given by connecting the wall-layers by the velocity in the middle, yielding a volume total flow rate of 

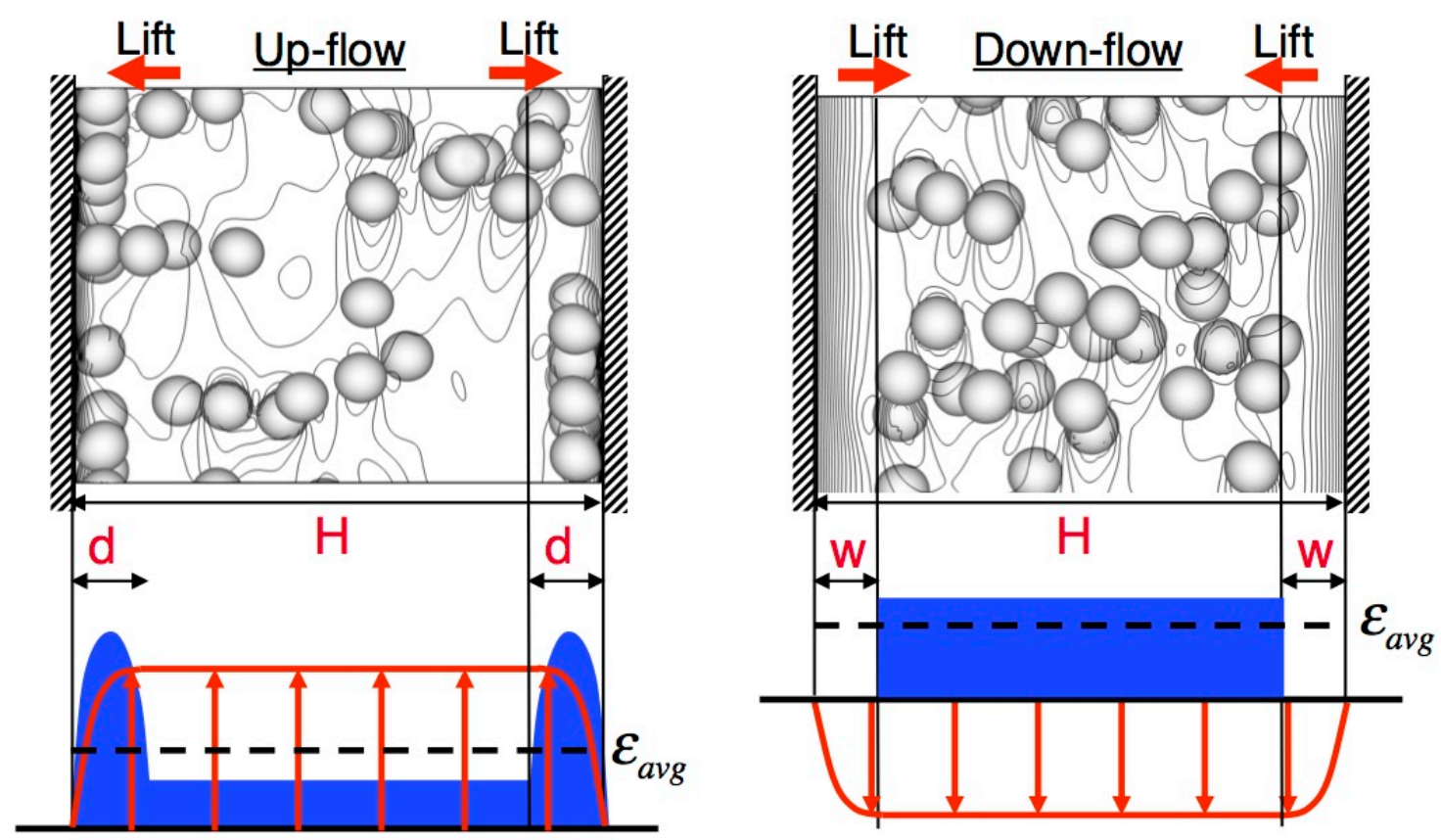

Fig. 1 A sketch of the structure of buoyancy driven bubbly flow in a vertical channel. In both cases the bubbles rise upward, but the direction of the fluid flow is different. In the frame on the left the flow is upward and in the frame on the right it is downward. The red profile shows the velocity and the blue region is the void fraction. Adopted from a similar figure in Lu et al. (2006).

$$
Q=-\frac{(\beta W)^{3}}{8 \mu\left(\beta+\varepsilon_{a v} g \Delta \rho\right)^{2}}\left[\frac{2}{3}-\varepsilon_{a v}+\frac{\Delta \rho g}{\beta}\left(1-\varepsilon_{a v}\right)\right]
$$

For turbulent flow the velocity profile is given by the law-of-the-wall, if the layer is sufficiently thick, but for thin wall-layers the flow is modified and becomes laminar for thin enough layers (Lu and Tryggvason, 2006).

For upflow, our simulations show that bubbles of the same size slide along the wall and the thickness of the layer is about one bubble diameter, $d_{b}$. Thus, knowing the average void fraction in the channel and the void fraction in the core region, the average void fraction in the wall-layer, or the number density of bubbles there, is easily found. Mass conservation for a channel with a bubbly wall-layer is,

$$
\varepsilon_{a v} W=\varepsilon_{c}\left(W-2 d_{b}\right)+\varepsilon_{w} 2 d_{b}
$$

and combining equations (14) and (6) gives the average void fraction in the wall-layer:

$$
\varepsilon_{w}=\varepsilon_{c}-\frac{W}{2 d_{b}} \frac{\beta}{g \Delta \rho}=\varepsilon_{a v}-\frac{\beta}{g \Delta \rho}\left(\frac{W}{2 d_{b}}-1\right) .
$$

Since $\beta<0$, the wall-layer void fraction is higher than the average void fraction. The velocity in the wall-layer is more complex than for the downflow case, because of the presence of the bubbles, which change the shape of the velocity profile and increase the wall shear, for a given velocity at the outer edge of the layer. In Fig. 1 the velocity profile and the void fraction distribution are shown schematically, along with the bubble distribution and a contour plot of the vertical velocity at one time, for both upflow and downflow.

As outlined above, DNS results for nearly spherical bubbles have lead to very simple analytical predictions for the void fraction profile for both upflow and downflow and for the velocity and the flow rate for downflow. Detailed comparisons of these formulas with DNS results show excellent agreement. For downflow we have also shown that the flow is relatively insensitive to the size of the bubbles as well as to the presence of bubbles of different sizes (Lu and Tryggvason 2007, 2008). Notice that the motion of the bubbles plays essentially no part in the discussions above, except in determining the density of the mixture. The relative velocity of the bubbles, their dispersion and the unsteady liquid motion depends, of course, on the void fraction and the shape of the bubbles, but these quantities are essentially decoupled from the average motion of the liquid.

Most of our studies of bubbly flows in vertical channels were done using relatively small domains and few bubbles, 


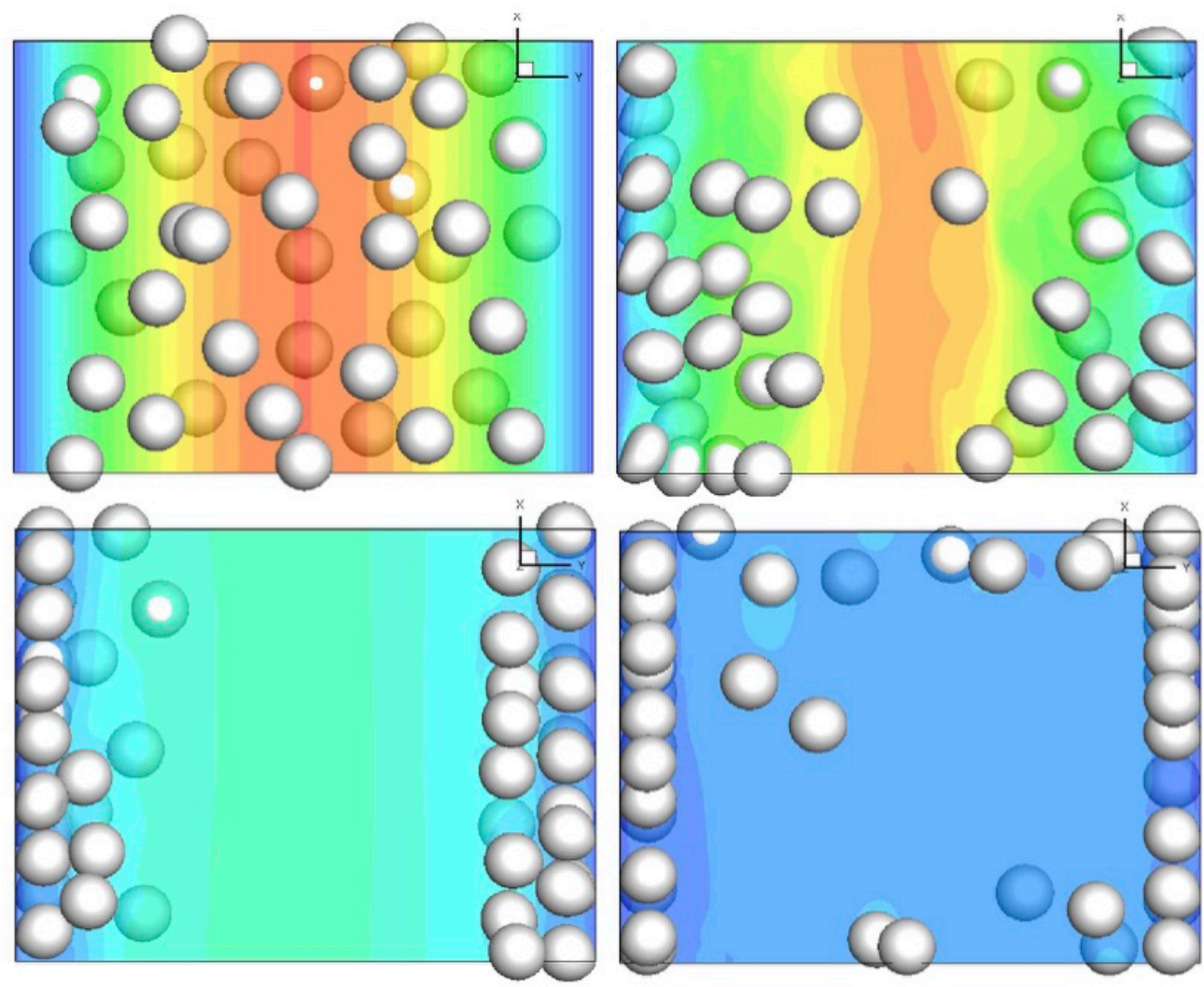

Fig. 2 The transient motion of laminar bubbly upflow in a vertical channel. The color shows the vertical velocity. The bubbles are first pushed to the walls and only after the flow has slowed down significantly do some of them return to the middle of the channel.

and for our simulations using initially turbulent flow the Reynolds number was modest. To examine what happens in larger systems, Lu and Tryggvason (2013) simulated over hundred bubbles in a turbulent channel flow with a friction Reynolds number of 250 . The overall flow structure at statistically steady state was found to be essentially the same as for the smaller systems, but it was also found that the bubbles in the wall-layer gathered together into a large cluster, so that part of each wall was covered with tightly packed bubbles and other regions had no bubbles. While exchange of bubbles between the core and the wall-layer is relatively rare, the clustering was found to increase the exchange rate slightly. The clustering of nearly spherical bubbles in upflow is very similar to the experimental observations of Takagi et al. $(2008,2011)$ who found that bubbles in a weak surfactant solution, where the strength of the surfactant was sufficient to prevent coalescence, but not strong enough to make the bubble surface immobile, formed wall-layers that included large clusters of bubbles.

The lift force experienced by bubbles depends sensitively on their deformability and it has been known for quite some time (Kariyasaki, 1987; Ervin and Tryggvason, 1997; Tomiyama et al., 2002) that the lift is greatly reduced and sometimes changes sign once the bubbles become deformable enough. We have examined this in some detail with simulations where we keep everything constant except the bubble deformability (by changing surface tension). The results show that in upflow the transition between bubbles that are nearly spherical and move to the walls and more deformable bubbles that move away from walls is rather abrupt (Dabiri et al., 2012). Furthermore, the flow structure at steady state is relatively insensitive to the value of the surface tension on either side of the transition (assuming though that for the deformable bubbles it is high enough to prevent breakup). The deformable bubbles do not accumulate at the walls and their effect on the flow rate, as compared to single-phase flow, tends to be relatively minimal, after the pressure gradient has been adjusted to account for the reduced mixture density due to the addition of the bubbles.

The focus of most of our studies of bubbles in channel flows has been on the statistically steady state and for large systems we have found it relatively difficult to get there. Indeed, for our largest simulations we did not go through the full transient but accelerated the convergence by a careful selection of the initial conditions and an adjustments of the 


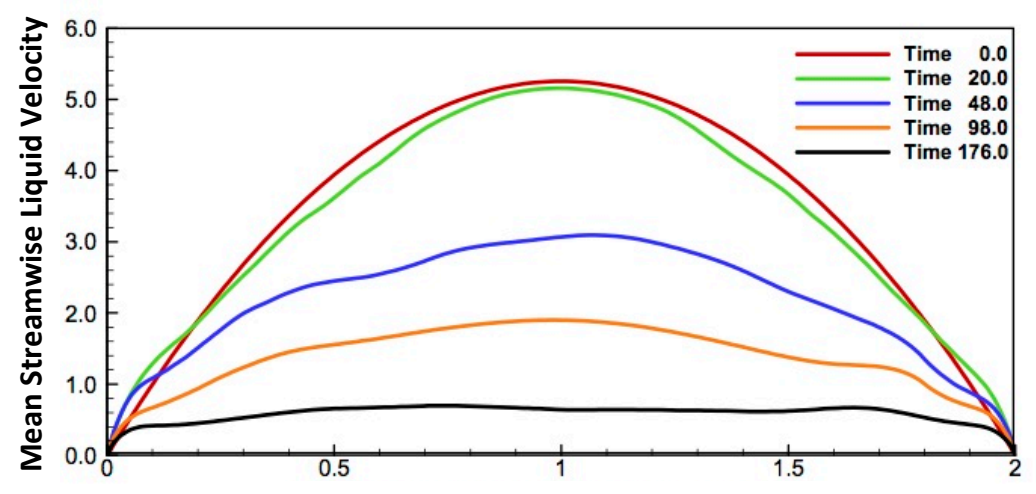

$X$ (Coordinate in wall-normal direction)

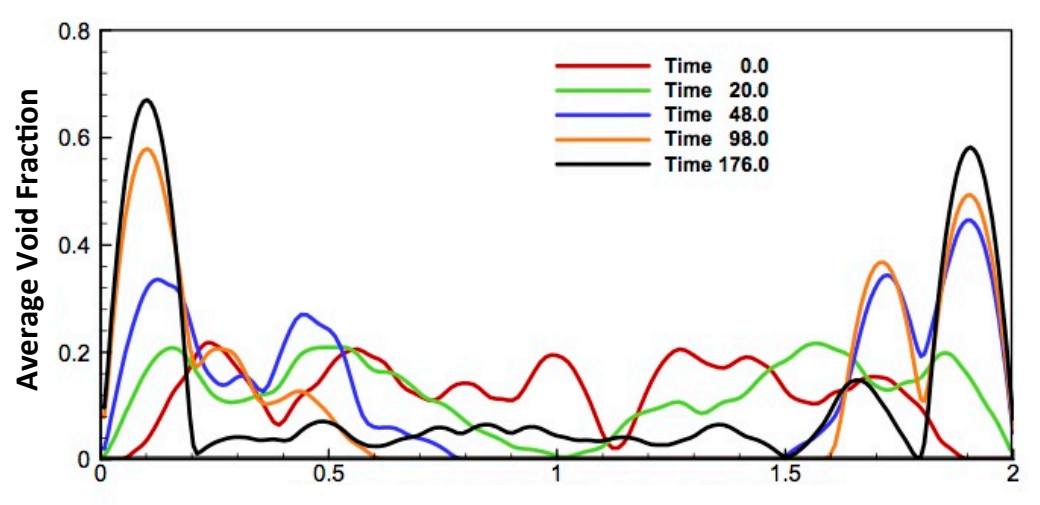

$X$ (Coordinate in wall-normal direction)

Fig. 3 The transient motion of laminar bubbly upflow in a vertical channel. The average vertical velocity (top) and the void fraction (bottom) are plotted versus the wall-normal coordinate for the simulation in Fig. 2 at five different times.

flow while it had not reached a steady state. This suggests that the transient is long and likely to be just as important as the steady state. To examine this we have done a few simulations where we inject bubbles into a laminar parabolic upflow and follow the evolution until it has reached an approximate steady state. The results show that the evolution is non-monotonic and takes place on at least two time scales. The flow is initially set up in such a way that the imposed pressure gradient and the weight of the mixture matches the wall shear stress. In the first stage of the evolution, essentially all the bubbles move to the walls, relatively quickly, and without much change in the liquid velocity. Once the bubbles are at the wall, the wall shear quickly increases and then the liquid starts to slowly decelerate. The reduction in the liquid velocity is usually very significant and takes a long time. Since the core region is nearly free of bubbles, its weight is not balanced by the imposed pressure gradient and its velocity eventually falls behind the velocity in the wall-layer, creating a slightly negative velocity gradient. This results in some of the bubbles moving out of the wall-layer, eventually reaching a balance where there are just enough bubbles in the core region for the weight of the mixture to balance the pressure gradient, and for the steady state results found earlier to apply. Results from one simulation of the transient motion of bubbly channel flows are shown in Figs. 2 and 3. Here, 44 bubbles of diameter 0.2 computational units are placed uniformly in a laminar channel flow, with an initial Reynolds number of 5256 . The material properties are selected to give a Morton number of $1.16 \times 10^{-9}$ and an Eötvös number of 0.167 . The size of the channel is $1.5 \times 2.0 \times 0.5$ computational units and it is resolved by a grid that has $192 \times 192 \times 64$ points, that are spaced uniformly in the stream-wise and span-wise direction but clustered in the wall-normal direction to give a finer resolution at the walls. The bubbles and the average vertical velocity are shown at four times in Fig. 2, where the color indicates the magnitude of the vertical velocity. The initial conditions are shown in the top left corner. In the second frame, top right corner, the bubbles are moving to the walls, but the velocity in the center is nearly unchanged. In the third frame essentially all the bubbles have moved to the walls and the velocity has been greatly reduced. Finally, some of the bubbles move back into the core in the forth frame as the velocity is reduced further. Figure 3 shows the average velocity in the top frame and the void fraction in the bottom frame, versus the wall-normal coordinate at five times, and 

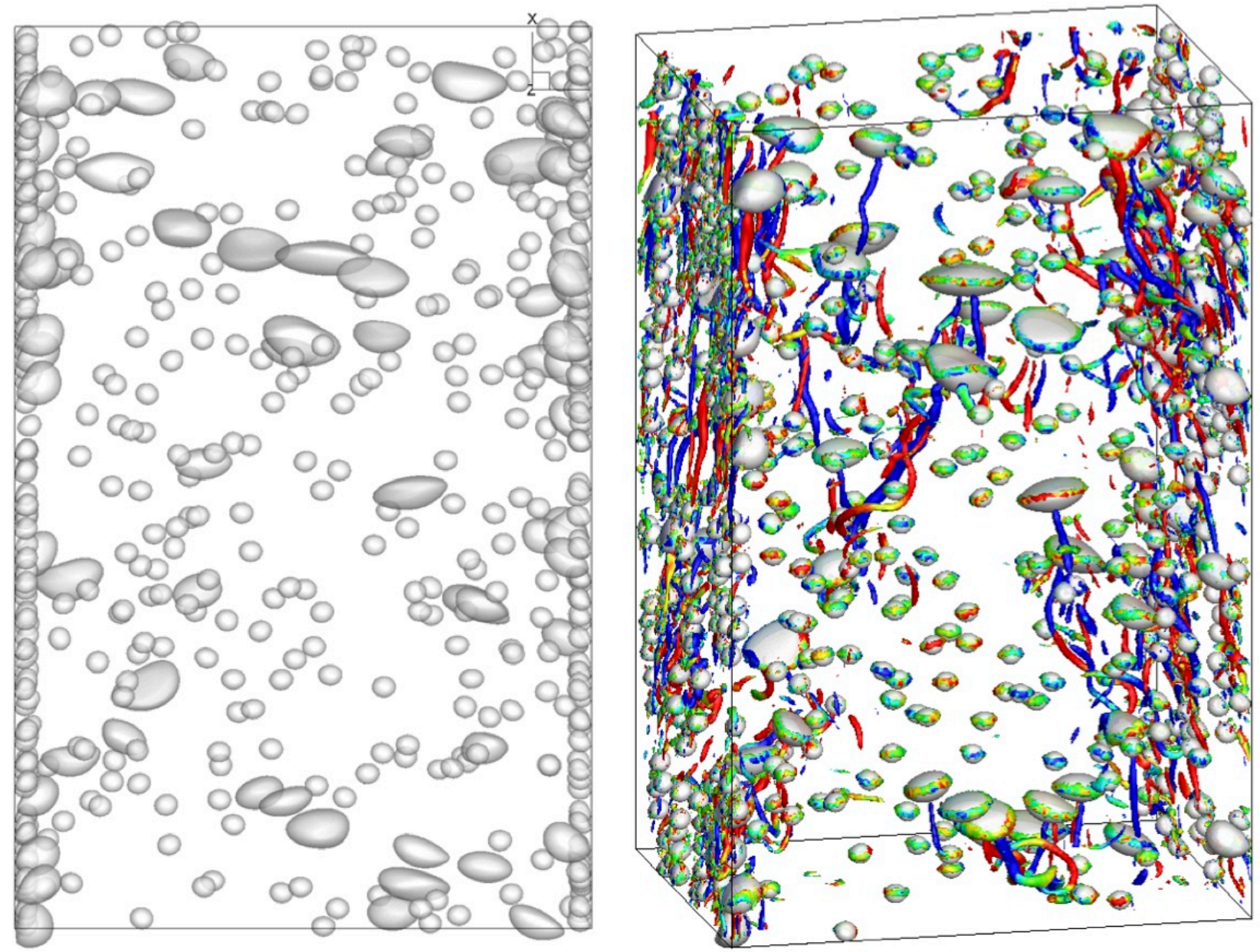

Fig. 4 The transient motion of turbulent bubbly upflow in a vertical channel at one time. The bubbles only are shown on the left and the bubbles plus the vorticity, visualized by the $\lambda_{2}$ method, on the right. The color indicates the orientation and rotational direction of the vortices.

it is again clear that while the velocity decreases monotonically, the evolution of the void fraction is more complex.

Most of the bubbly systems studied so far have been relatively simple and it is important to recognize that experimental and industrial systems are in most cases more complex. The bubbles are, in particular, likely to come in different sizes and the Reynolds number is usually higher. To examine the effect of a distribution of bubble sizes, as well as larger Reynolds numbers, we have examined the initial evolution of several hundred bubbles of different sizes, where the smallest ones remain nearly spherical and will move to the walls and the largest ones are much more deformable and are likely to stay in the center of the channel. The domain size is $2 \pi \times 4 \times \pi$ in the stream-wise, wall-normal and span-wise direction, respectively, resolved by $1024 \times 768 \times 512$ grid points and run using 2048 processors on the Titan supercomputer at the Oak Ridge National Laboratory. The physical parameters are selected such that the Morton number is $5.75 \times 10^{-10}$ and the void fraction is 0.0304 . The bubbles come in four sizes. The majority of them, 504 total, are small, with a diameter equal to 0.16 in computational units, but then we add 50 bubbles with a diameter of 0.306 , 13 with a diameter of 0.386 , and 4 large bubbles with a diameter of 0.44 . The corresponding Eötvös numbers are 0.5 , 1.83, 2.9 and 3.78. The results of a study by Dabiri et al. (2012) suggest that the transition between bubbles pushed to the wall and those that are not is around Eo equal to 2.5, so we expect the smallest two bubble groups to accumulate at the walls. The numbers of bubbles in each group was selected so that there are enough small bubbles that can be pushed to the wall to put the core in hydrostatic equilibrium. The properties of the fluid and the bubbles are the same as in our earlier simulations (Lu and Tryggvason, 2013), but the domain size is eight times larger, giving a friction Reynolds number of $\mathrm{Re}^{+}=500$. The bubbles are initially distributed nearly uniformly across the domain but as they start to rise, 
the smaller bubbles start to migrate toward the walls and form dense wall-layers. As discussed above, the steady state for channels with spherical bubbles, where the lift force pushes the bubbles toward the wall and a bubbly wall-layer is formed, consists of wall-layers and a homogeneous core region where the number of bubbles is such that the weight of the mixture balances the imposed pressure gradient. Given our experience with the transient evolution of laminar flows, we expect most of the nearly spherical bubbles to initially move to the walls, while the larger deformable ones stay in the middle of the channel. The flow is shown at a relatively early time in Fig. 4, where the small bubbles have migrated to the walls but the average velocity is nearly unchanged from the initial conditions. In the frame on the left the bubbles are shown in a view parallel to the walls. A significant fraction of the small bubbles have moved to the walls, along with some of the slightly larger ones but the largest bubbles remain in the center of the channel. In the frame on the right the bubbles and the vortical structures in the flow, as visualized by the $\lambda_{2}$ method, are shown in a three-dimensional view. The vortices are color coded according to their orientation, with red and blue vortices being aligned with the flow, but with opposite rotation, and green and yellow ones having an orientation perpendicular to the flow direction. A comparison of this frame with earlier times shows that the coherence of the vorticity next to the walls has been disrupted significantly with the arrival of the small bubbles, although large longitudinal vortices can be seen in regions that are free of bubbles. Relatively coherent vortices aligned with the flow are also seen in the middle of the channel, behind the bubbles. A careful inspection of the figure also shows that the bubbles are not uniformly distributed at the wall but have started to form clusters, leaving some parts of the wall without bubbles. This is a similar evolution as seen by Lu and Tryggvason (2013).

\section{Complex Bubbly Flows}

Done correctly, DNS yield an essentially exact solution of well-defined and validated equations. Thus, they allow us to examine systems that often cannot be set up experimentally (monodisperse clean bubbles, for example), or explore parameter variations in ranges that cannot be reached in the laboratory (change surface tension but keep bubble size the same and the interface clean, for example). Often, however, we are interested in how the behavior of the flow changes if we add more complex physics, or how other physical processes behave in the presence of bubbles.

Real bubbles are rarely clean and it is well known that surfactants, or contamination, change the behavior of a single bubble significantly. The effect on the collective motion of bubbles in channel flows are also significant as the experimental observations of Takagi et al. (2008) show. To examine how surfactants change the evolution we have extended the front tracking method used for most of our simulations to account for miscible surfactants (Muradoglu and Tryggvason, 2008 and 2014). The results, which are confined to the motion of a single bubble so far, show that the presence of a surfactant can have a significant influence on the motion of the bubble and its interaction with a wall. A sufficiently strong contaminate can, in particular, reverse the lift on nearly spherical bubbles so that a bubble in upflow will move away from the wall instead of toward it, Thus, we would not expect wall-layers for sufficiently strong surfactants. A DNS study of the collective dynamics of many contaminated bubbles remains to be done.

In order for DNS to have an impact on our current understanding of flows in bubble columns and other practical applications, it is not sufficient to examine just the bubble motion. The transfer of heat and mass, as well as chemical reactions must be included. The addition of an equation for the temperature distribution is relatively straightforward but relatively little has, however, been done to use DNS to explore heat transfer in bubbly flows. Deen and Kuipers (2013) showed that the passage of a single bubble, or a small group of bubbles, generally increases the local heat transfer. Tanaka (2011) and Dabiri and Tryggvason (2015) examined many bubbles in turbulent channel flows and showed that the bubbles enhances the overall heat transfer, both for nearly spherical bubbles that form wall-layers, as well as for more deformable ones that stay away from the walls. Mass transfer and reactions are in principle relatively simple. We need to solve additional advection/diffusion equations that include source terms when there are reactions. In reality, however, the large range of scales, due to low mass diffusivities and fast reactions, introduce significant challenges.

In some cases we can assume that the chemistry in bubble columns is dominated by two processes. The gas in the bubbles diffuses into the liquid and reacts with the gas already dissolved there and then the product reacts with the gas diffusing from the bubbles, resulting in an undesirable byproduct. The goal is to maximize the first process and minimize the second. Raffensberger et al. (2003) showed that for stationary bubbles the results are strongly dependent on the details of the flow, and a large deformable bubble with a stationary wake can, for example, trap products from the first (desirable) process in the wake and greatly increase their ability to react with gas from the bubble (forming the 
undesirable by-product). Koynov et al. (2005) showed that the sensitivity carries over to flows with freely moving bubbles but the exact dependency on the governing parameters is even more complex. Other investigations of mass transfer include Davidson and Rudman (2002), Bothe et al. (2011) who also looked at simple reactions, Zhang et al. (2006) who modeled a soluble surfactant, and a study of the hydrogenation of nitroarenes by Radl et al. (2008).

Although simulations of flows with heat and mass transfer involve, at least the simplest cases when the bubbly flow is not affected by the temperature or the mass concentration, just solving additional advection/diffusion equations, the diffusion of mass in liquids, in particular, is much slower than the diffusion of momentum and this leads to significant disparity in the scales that need to be resolved. Mass boundary layers at bubble surfaces are, in particular, very thin. Thus, the simulations of Koynov et al. (2005) and Radl et al. (2008) were limited to two-dimensional flow only and the grid used to resolve the mass transfer was much finer than what was used for the fluid flow. The structure of the mass boundary layer is, however, relatively simple and that motivated Aboulhasanzadeh et al. (2012) to introduce a numerical strategy where the boundary layer was resolved by a semi-analytical model, developed using a boundary layer approximation, to capture mass diffusing out from the bubble. At the back of the bubbles, where the mass boundary layer became thick, the mass was transferred to the fixed grid and evolved by solving an advection/diffusion equation on the regular stationary grid. The accuracy of the approach was demonstrated by comparisons with fully resolved simulations of two-dimensional flows. Subsequent comparisons with experimental results in Aboulhasanzadeh et al. (2013) showed excellent agreement and the method was then applied to examine how bubble interactions influenced mass transfer (Aboulhasanzadeh and Tryggvason, 2014). The results were compared with predictions by a simple cell model where the bubbles remain fixed in a periodic array, and showed that although the interactions increased mass transfer, the freely evolving bubbles are also slowed down, which decreases the mass transfer. Thus, the overall mass transfer does not change much for the particular parameter range examined.

\section{Conclusions}

DNS of bubbly multiphase flows have come a long way in the last ten years or so. Simulations of hundreds of bubbles in turbulent channel flows, for long enough times so that meaningful statistics can be collected, are now possible. The data produced by such simulations is already leading to significant new insight, but the possibility of using the results to help with the development of reduced order descriptions of the flow, as closure models for either two-fluid equations for the average flow or large eddy like models is perhaps the most exciting outcome. For single phase flows DNS have lead to major improvements in modeling and, in particular, the development of models where the large scale flows are resolved and the smaller scales are modeled. From a purist point of view it may be inaccurate to refer to many of those simulations as Large Eddy Simulations (LES), since the range of unresolved scales is often relatively large, so we prefer to call them LES-like, but the reality is that these simulations are increasingly finding use in engineering design. Similar development is needed for multiphase flows and, as for single-phase flows, it is likely that the availability of DNS data will lead to both new modeling strategies and new ways to close the resulting equations. Given the complexity of gas-liquid flows in particular, where the flow can transition between different regimes that behave in fundamentally different ways, a reduced order description is likely to be different from what has worked for single-phase flows. The number of variables that one needs to keep track of is likely to be larger, since some information about the interface topology are needed, and the range of parameters is larger. Finding complex relationships between a large numbers of variables is likely to require a more sophisticated data processing than has been used in the past, and it seems certain that methods collectively referred to as statistical learning will be increasingly important in the processing of DNS data. Not only should such techniques help find complex relationships, but a statistical approach to the processing will also allow a quantification of the uncertainty. We have recently experimented with using neural networks to find the dependency of closure terms for a simple averaged model of bubbly flows in simple situations, where there are no walls and it seems likely that the closure terms (the lateral gas flux and the streaming stresses) depend only on the resolved average quantities. The results are promising, but this approach needs to be explored further in the context of both more complex flows and more sophisticated models. Further development is, in particular, needed for LES-like models, where some scales are resolved and other are not. Attempts to develop such models for multiphase flows are currently mostly limited to simple disperse flows. A group in France has started to explore LES-like modeling of more complex multiphase flows, both by deriving filtered models and using DNS data to evaluate the closure terms (Toutant et al. 2008 and 2009, for example). This seems like a 
promising start but much more remains to be done. Perhaps the biggest unknown in modeling of multiphase flows is the question of what are the appropriate statistical quantities that are needed to describe the average state of the unresolved flow. For single phase flow the turbulent kinetic energy is used in many models to quantify the intensity of the unresolved turbulence and the scale of the turbulent motion is described by the turbulent dissipation rate or other similar quantity. For all but the simplest multiphase flows we need information about the phase distribution in addition to the average void fraction. The area density has been used in the past but most likely we will need to know much more about the average topology. The mostly monodisperse disperse bubbly flows examined so far are likely to be among the simpler ones when it comes to modeling, but they are a natural starting point. If we cannot accurately model those, it is unlikely that flows with many bubbles of different sizes and complex churn turbulent flows undergoing repeated topology changes can be dealt with.

As the ability to examine the collective motion of many bubbles grows, the needs to examine problems with more complex physics will also become more urgent. As discussed above, efforts to include mass and heat transfer, as well as reactions and phase change, are emerging, but much remains to be done and we believe, in particular, that modeling strategies similar to the one we used for mass transfer in Aboulhasanzadeh et al. (2012) are likely to play a much larger role, including for the accurate modeling of topology changes. To many researchers, DNS means fully resolving every continuum temporal and spatial length and time scale for systems that are large enough to include non-trivial scale interactions and for long enough time so that converged statistical quantities can be collected. When some of the small scales are treated differently than the larger scales (such as in the multiscale approach discussed by Tomiyama, 1998) one can argue that we do not really have a DNS, but our contention is that as our problems become more complex, our numerical strategies must allow for more sophisticated approaches. As additional physical processes are incorporated into our mathematical models, we are also likely to be faced with increased uncertainty in the mathematical models used to describe the new physics, rendering traditional DNS less applicable.

\section{ACKNOWLEDGEMENT}

Our recent studies of bubbly flows have been supported by the Consortium for Advanced Simulation of Light Water Reactors (CASL). This research used resources of the Oak Ridge Leadership Computing Facility at the Oak Ridge National Laboratory, which is supported by the Office of Science of the U.S. Department of Energy under Contract No. DE-AC05-00OR22725.

\section{References}

Aboulhasanzadeh, B. and Tryggvason, G., Effect of bubble interactions on mass transfer in bubbly flow, International Journal of Heat and Mass Transfer, Vol. 79 (2014), pp. 390-396.

Aboulhasanzadeh, B., Thomas, S., Taeibi-Rahni, M. and Tryggvason, G., Multiscale computations of mass transfer from buoyant bubbles, Chemical Engineering Science, Vol. 75 (2012) pp. 456-467.

Aboulhasanzadeh, B., Hosoda, S., Tomiyama, A. and Tryggvason, G., A validation of an embedded analytical description approach for the computations of high Schmidt number mass transfer from bubbles in liquids, Chemical Engineering Science, Vol. 101 (2013) pp. 165-174.

Antal, S.P., Lahey, R.T. and Flaherty, J.E., Analysis of phase distribution in fully developed laminar bubbly two-phase flows, International Journal of Multiphase Flow, Vol. 15 (1991), pp. 635-652.

Azpitarte, O.E. and Buscaglia, G.C., Analytical and numerical evaluation of two-fluid model solutions for laminar fully developed bubbly two-phase flows, Chemical Engineering Science, Vol. 58 (2003), pp. 3765-3776.

Brackbill, J.U., Kothe, D.B. and Zemach, C., A continuum method for modeling surface tension, Journal of Computational Physics, Vol. 100 (1992), pp. 335-354.

Bothe, D. and Fleckenstein S., A volume-of-fluid-based method for mass transfer processes at fluid particles, Chemical Engineering Science, Vol. 101 (2013), pp. 283-302.

Bothe, D., Kröger, M., Warnecke, H.J., A VOF-based conservative method for the simulation of reactive mass transfer from rising bubbles, Fluid Dynamics \& Materials Processing, Vol. 7 (2011), pp. 303-316.

Bunner, B. and Tryggvason, G., Dynamics of homogeneous bubbly flows. Part 1. Rise velocity and microstructure of the bubbles, Journal of Fluid Mechanics, Vol. 466 (2002a), pp. 17-52.

Bunner, B. and Tryggvason, G., Dynamics of homogeneous bubbly flows. Part 2. Velocity fluctuations, Journal of 
Fluid Mechanics, Vol. 466 (2002b), pp. 53-84.

Bunner, B. and Tryggvason, G., Effect of bubble deformation on the stability and properties of bubbly flows, Journal of Fluid Mechanics, Vol. 495 (2003), pp. 77-118.

Celik, I. and Gel, A., A new approach in modeling phase distribution in fully developed bubbly pipe flow, Flow, Turbulence and Combustion, Vol. 68 (2002), pp. 289-311.

Dabiri, S., Lu, J., and Tryggvason, G., Transition between regimes of a vertical channel bubbly upflow due to bubble deformability, Physics of Fluids, Vol. 25 (2013), 102110.

Dabiri, S. and Tryggvason, G., Heat transfer in turbulent bubbly flow in vertical channels, Chemical Engineering Science. Vol. 122 (2015), pp. 106-113.

Davidson, M.R., and Rudman, M., Volume-of-fluid calculation of heat or mass transfer across deforming interfaces in two-fluid flow, Numerical Heat Transfer, Part B: Fundamentals, Vol. 41 (2002), pp. 291-308.

Deckwer, W.-D., Bubble Column Reactors (1992), Wiley.

Deen, N.G. and Kuipers, J.A.M., Direct numerical simulation of wall-to liquid heat transfer in dispersed gas-liquid two-phase flow using a volume of fluid approach, Chemical Engineering Science, Vol. 102 (2013), pp. $268-282$.

Descamps, M.N., Oliemans, R.V.A., Ooms, G. and Mudde, R.F., Air-water flow in a vertical pipe: experimental study of air bubbles in the vicinity of the wall, Experiments in Fluids, Vol. 45 (2008), pp. 357-370.

Dijkhuizen, W., Roghair, I., van Sint Annaland, M. and Kuipers, J.A.M., DNS of gas bubbles behaviour using an improved 3d front tracking model-drag force on isolated bubbles and comparison with experiments, Chemical Engineering Science, Vol. 65 (2010a), pp. 1415-1426.

Dijkhuizen, W., Roghair, I., van Sint Annaland, M. and Kuipers, J.A.M., DNS of gas bubbles behaviour using an improved 3d front tracking model-model development, Chemical Engineering Science, Vol. 65 (2010b), pp. $1427-1437$.

Drew, D.A. and Lahey, Jr., R.T., Analytical modeling of multiphase flow. In: Particulate Two-phase Flow (1993), pp. 509-566. Butterworth-Heinemann.

Drew, D.A. and Passman, S.L. Theory of multicomponent fluids (1999), Springer.

Ervin, E.A. and Tryggvason, G., The rise of bubbles in a vertical shear flow, Journal of Fluids Engineering, Vol. 119 (1997), pp. 443-449.

Esmaeeli, A. and Tryggvason, G., Direct numerical simulations of bubbly flows. Part I. Low Reynolds number arrays, Journal of Fluid Mechanics, Vol. 377 (1998), pp. 313-345.

Esmaeeli, A. and Tryggvason, G., Direct numerical simulations of bubbly flows. Part II. Moderate Reynolds number arrays, Journal of Fluid Mechanics, Vol. 385 (1999), pp. 325-358.

Esmaeeli, A. and Tryggvason, G., A direct numerical simulation study of the buoyant rise of bubbles at $\mathrm{O}(100)$ Reynolds number, Physics of Fluids, Vol. 17 (2005), 093303.

Figueroa-Espinoza, B. and Zenit, R., Clustering in high Re monodispersed bubbly flows, Physics of Fluids, Vol. 17 (2005), 091701.

Furusaki, S., Fan, L.-S. and Garside, J., The Expanding World of Chemical Engineering (2nd ed). Taylor and Francis, 2001.

Guet, S., Ooms, G. and Oliemans, R.V.A., Simplified two-fluid model for gas-lift efficiency predictions, AIChE Journal, Vol. 51 (2005), pp. 1885-1896.

Guet, S., Ooms, G., Oliemans, R.V.A. and Mudde, R.F., Bubble size effect on low liquid input drift-flux parameters, Chemical Engineering Science, Vol. 59 (2004), pp. 3315-3329.

Hao, Y. and Prosperetti, A., A numerical method for three-dimensional gas-liquid flow computations, Journal of Computational Physics, Vol. 196 (2004), pp. 126-144.

Harlow, F.H. and Welch, J.E., Numerical calculation of time-dependent viscous incompressible flow of fluid with a free surface, Physics of Fluids, Vol. 8 (1965), pp. 2182-2189.

Hayashi, K., and Tomiyama, A., Interface tracking simulation of mass transfer from a dissolving bubble, Journal of Computational Multiphase Flows, Vol. 3 (2011), pp. 247-262.

Hirt, C.W. and Nichols, B.D., Volume of Fluid (VOF) method for the dynamics of free boundaries, Journal of Computational Physics, Vol. 39 (1981), pp. 201-226.

Hua, J. and Lou, J., Numerical simulation of bubble rising in viscous liquid, Journal of Computational Physics, Vol. 222 (2007), pp. 769-795. 
Kariyasaki, A. Behavior of a gas bubble in a liquid flow with a linear velocity profile. Transactions of Japan Society of Mechanical Engineers, Series B, Vol. 53 (1987), pp. 744-749 (in Japanese)

Kashinsky, O.N. and Randin, V.V., Downward bubbly gas-liquid flow in a vertical pipe, International Journal of Multiphase Flow, Vol. 25 (1999), pp. 109-138.

Koynov, A., Khinast, J.G. and Tryggvason, G., Mass transfer and chemical reactions in bubble swarms with dynamic interfaces, AIChE Journal, Vol. 51 (2005), pp. 2786-2800.

Kuo, T.C., Pan, C. and Chieng, C.C., Eulerian-Lagrangian computations on phase distribution of two-phase bubbly flows, International Journal for Numerical Methods in Fluids, Vol. 24 (1997), pp. 579-593.

Liu, T.J., Investigation of the wall shear stress in vertical bubbly flow under different bubble size conditions, International Journal of Multiphase Flow, Vol. 23 (1997), pp. 1085-1109.

Liu, T.J. and Bankoff, S.G., Structure of air-water bubbly flow in a vertical pipe-I. liquid mean velocity and turbulence measurements, International Journal of Heat and Mass Transfer, Vol. 36 (1993), pp. 1049-1060.

Lopez de Bertodano, M., Lahey Jr., R.T. and Jones, O.C., Development of a k- $\varepsilon$ model for bubbly two-phase flow, Journal of Fluids Engineering, Vol. 13 (1987), pp. 327-343.

Lopez de Bertodano, M., Lahey Jr., R.T. and Jones, O.C., Phase distribution in bubbly two-phase flow in vertical ducts. International Journal of Multiphase Flow, Vol. 20 (1994), pp. 805-818.

Lu, J., Biswas, S. and Tryggvason, G., A DNS study of laminar bubbly flows in a vertical channel, International Journal of Multiphase Flow, Vol. 32 (2006), pp. 643-660.

Lu, J. and Tryggvason, G., Numerical study of turbulent bubbly downflows in a vertical channel, Physics of Fluids, Vol. 18 (2006), 103302.

Lu, J. and Tryggvason, G., Effect of bubble size in turbulent bubbly downflow in a vertical channel, Chemical Engineering Science, Vol. 62 (2007), pp. 3008-3018.

Lu, J. and Tryggvason, G., Effect of bubble deformability in turbulent bubbly upflow in a vertical channel, Physics of Fluids, Vol. 20 (2008), 040701.

Lu, J. and Tryggvason, G., Dynamics of nearly spherical bubbles in a turbulent channel upflow, Journal of Fluid Mechanics, Vol. 732 (2013), pp. 166-189.

Matos, A., de Rosa, E.S. and Franca, F.A. The phase distribution of upward co-current bubbly flows in a vertical square channel, Journal of the Brazilian Society of Mechanical Sciences and Engineering, Vol. 26 (2004), pp. 308-316.

Mendez-Diaz, S., Zenit, R., Chiva, S., Munoz-Cobo, J.L. and Martinez-Martinez, S., A criterion for the transition from wall to core peak gas volume fraction distributions in bubbly flows, International Journal of Multiphase Flow, Vol. 43 (2012), pp. 56-61.

Molin, D., Marchioli, C. and Soldati, A., Turbulence modulation and microbubble dynamics in vertical channel flow. International Journal of Multiphase Flow, Vol. 42 (2012), pp. 80-95.

Muradoglu, M. and Kayaalp, A. D., An auxiliary grid method for computations of multiphase flows in complex geometries, Journal of Computational Physics, Vol. 214 (2006), pp. 858-877.

Muradoglu, M., and Tryggvason, G., A front-tracking method for computation of interfacial flows with soluble surfactants, Journal of Computational Physics, Vol. 227 (2008), pp. 2238- 2262.

Muradoglu, M., and Tryggvason, G., Simulations of soluble Surfactants in 3D multiphase flow, Journal of Computational Physics, Vol. 274 (2014), pp. 737-757.

Nakoryakov, V.E., Kashinsky, O.N., Randin, V.V. and Timkin, L.S., Gas-liquid bubbly flow in vertical pipes, Journal of Fluids Engineering, Vol. 118 (1996), pp. 377-382.

Politano, M.S., Carrica, P.M. and Converti, J. A model for turbulent polydisperse two-phase flow in vertical channel, International Journal of Multiphase Flow, Vol. 29 (2003), pp. 1153-1182.

Radl, S., Koynov, A., Tryggvason, G., and Khinast, J.G., DNS-based prediction of the selectivity of fast multiphase reactions: Hydrogenation of nitroarenes, Chemical Engineering Science, Vol. 63 (2008) pp. 3279-3291.

Raffensberger, J., Koynov, A.A., Glasser, B.J. and Khinast, J.G., Influence of particle properties on the yield and selectivity of fast heterogeneously catalyzed gas- liquid reactions, International Journal of Chemical Reactor Engineering, Vol. 1 (2003), A15.

Sankaranarayanan, K., Kevrekidis, I.G., Sundaresan, S., Lu, J. and Tryggvason, G., A comparative study of lattice Boltzmann and front-tracking finite-difference methods for bubble simulations. International Journal of Multiphase Flow, Vol. 29 (2003), pp. 109-116. 
Serizawa, A., Kataoka, I. and Michiyoshi, I., Turbulence structure of air-water bubbly flow-II. Local properties, International Journal of Multiphase Flow, Vol. 2 (1975a), pp. 235-246.

Serizawa, A., Kataoka, I. and Michiyoshi, I., Turbulence structure of air-water bubbly flow-III. Transport properties, International Journal of Multiphase Flow, Vol. 2 (1975b), pp. 247-259.

van Sint Annaland, M., Dijkhuizen, W., Deen, N.G. and Kuipers, J.A.M., Numerical simulation of gas bubbles behaviour using a 3D front tracking method, AIChE Journal, Vol. 52 (2006), pp. 99-110.

So, S., Morikita, Takagi, S. and Matsumoto, Y., Laser doppler velocimetry measurement of turbulent bubbly channel flow, Experiments in Fluids, Vol. 33 (2002), pp. 135-142.

Sussman, M., Smereka, P. and S. Osher, S., A level set approach for computing solutions to incompressible two-phase flows, Journal of Computational Physics, Vol. 114 (1994), pp. 146-159.

Takagi, S. and Matsumoto, Y., Surfactant effects on bubble motion and bubbly flows, Annual Reviews of Fluid Mechanics, Vol. 43 (2011), pp. 615-636.

Takagi, S., Ogasawara, T. and Matsumoto, Y., The effects of surfactant on the multiscale structure of bubbly flows, Philosophical Transactions of the Royal Society A, Vol. 366 (2008), pp. 2117-2129.

Tanaka, M., Numerical study on flow structures and heat transfer characteristics of turbulent bubbly upflow in a vertical channel. In: Zhu, J. (Ed.), Computational Simulations and Applications (2011), pp. 119-142. In Tech, Rijeka, Croatia,

Tomiyama, A. Struggle with computational bubble dynamics. Multiphase Science and Technology Vol. 10 (1998), pp. $369-405$.

Tomiyama, A., Tamai, H., Zun, I. and Hosokawa, S., Transverse migration of single bubbles in simple shear flows, Chemical Engineering Science, Vol. 57 (2002), pp. 1849-1858.

Tryggvason, G., Bunner, B., Esmaeeli, A., Juric, D., Al-Rawahi, N., Tauber, W., Han, J., Nas, S. and Jan, Y.-J., A front tracking method for the computations of multiphase flow, Journal of Computational Physics, Vol. 169 (2001), pp. $708-759$.

Tryggvason, G., Scardovelli, R. and Zaleski S., Direct Numerical Simulations of Gas-Liquid Multiphase Flow (2011), Cambridge University Press.

Toutant, A., Labourasse, E., Lebaigue, O., and Simonin, O., DNS of the interaction between a deformable buoyant bubble and spatially decaying turbulence: a priori tests for LES two-phase flow modeling, Computers and Fluids, Vol. 37 (2008), pp. 877-886.

Toutant, A., Chandesris, M., Jamet, D. and Lebaigue, O., Jump conditions for filtered quantities at an under-resolved interface. Part 2: A priori tests, International Journal of Multiphase Flow, Vol. 35 (2009), pp. 1119-1129.

Unverdi, S. O. and Tryggvason, G., A front-tracking method for viscous, incompressible, multi-fluid flows, Journal of Computational Physics, Vol. 100 (1992), pp. 25-37.

Wang, S. K., Lee, S.J., Jones Jr., O.C. and Lahey Jr., R.T., 3-D turbulence structure and phase distribution in bubbly two-phase flows, International Journal of Multiphase Flow, Vol. 13 (1987), pp. 327-343.

Youngs. D. L., Time dependent multimaterial flow with large fluid distortion. In K. M. Morton and M. J. Baines, editors, Numerical methods for fluid dynamics (1982), pp. 27-39, Academic Press, New York.

Zhang, J., Eckmann, D.M. and Ayyaswamy, P.S., A front tracking method for a deformable intravascular bubble in a tube with soluble surfactant transport, Journal of Computational Physics, Vol. 214 (2006), pp. 366-396.

Zenit, R., Tsang, Y.H., Koch, D.L., and Sangani, A.S., Shear flow of a suspension of bubbles rising in an inclined channel, Journal of Fluid Mechanics, (2004), Vol. 515, pp. 261-292. 